\title{
Medical Image of the Week: Focal Myopericarditis
}

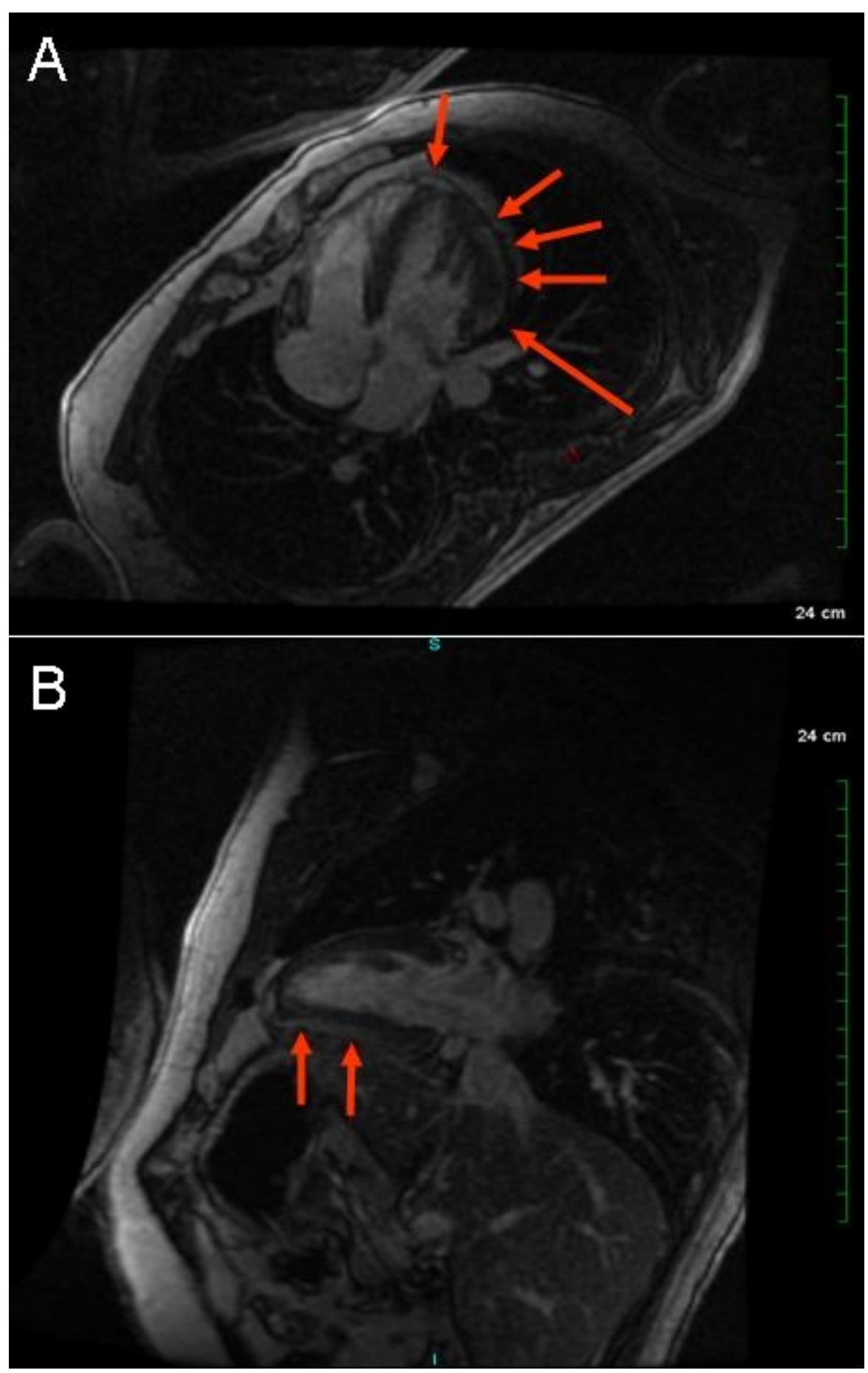

Figure 1. Transverse view (panel A) and longitudinal view (panel B) of MRI with gadolinium enhanced contrast of chest showing abnormal enhancement at the level of

the cardiac apex and also at the mid aspect of the infero-lateral wall and near the cardiac base within the lateral wall, consistent with the clinically suspected diagnosis of focal myopericarditis. 
A 44-year-old man with no significant past medical history was admitted with a history of two episodes of substernal chest pain unrelated to exertion which had resolved spontaneously. Admission vital signs were within normal limits and physical examination was unremarkable. Basic lab tests were normal and urine toxicology was negative. Electrocardiogram was unremarkable with no ST/T changes. Troponin I was elevated at 4.19 which trended up to 6.57. An urgent cardiac angiogram was done which revealed normal patent coronaries. His transthoracic echocardiogram was also reported to be normal. He continued to have intermittent episodes of chest pain that was partially relieved by morphine. Erythrocyte sedimentation rate and C-reactive protein were elevated. Work up for autoimmune diseases, vasculitis, myocarditis panel were insignificant. Later, magnetic resonance imaging (MRI) with gadolinium enhanced contrast (Figure 1) was obtained which showed abnormal epicardial/subepicardial myocardial enhancement within the inferolateral wall and cardiac apex consistent with focal myopericarditis. He was started on Ibuprofen and colchicine. His chest pain significantly improved and he is currently following up as an outpatient.

Acute myo-pericarditis is primarily a pericarditic syndrome with variable myocardial involvement which is usually seen in male adolescents $(1,2)$. There are 3 main etiologic categories: idiopathic, infectious or immune mediated. Patients present with chest pain that is sharp in nature with gradual onset radiating to the interscapular region, increasing with inspiration and easing with leaning forward. Pericardial friction rub on physical exam is considered pathognomonic. A typical pattern of ECG evolution includes diffuse ST segment elevation and PR depression, followed by normalization of ST and PR segments and then diffuse T wave inversion. Troponin I levels may be elevated, provides a rough estimate of the extent of myocardial inflammation. Cardiac MRI with gadolinium contrast is the best imaging modality to define the extent of myocardial involvement and patency of coronary system which is not always readily available. Therapy of choice is aspirin (1-1.5 g/day as mean dose) or nonsteroidal antiinflammatory drugs such as ibuprofen for 7-10 days until symptom resolution. Colchicine should be the initial therapy in all refractory cases and in recurrent pericarditis. Physical exercise is contraindicated for at least 6 months from the onset of illness.

Chandramohan Meenakshisundaram MD, Nanditha Malakkla MD, and Venu Ganipisetti MD.

Department of Internal Medicine, Presence Saint Francis Hospital Evanston, IL USA

\section{References}

1. Imazio M, Cooper LT. Management of myopericarditis. Expert Rev Cardiovasc Ther. 2013;11(2):193-201. [CrossRef] [PubMed]

2. Sharma J, Fernandes N, Alvarez D, Khanna S.Acute myopericarditis in an adolescent mimicking acute myocardial infarction. Pediatr Emerg Care. 2015;31(6):427-30. [CrossRef] [PubMed] 